\title{
Descentralización educativa y comunidades autónomas: el caso andaluz (1982-1986)
}

\author{
Educational decentralization and regions: the Andalusian case
} (1982-1986)

\author{
Manuel Hijano del Río \\ Universidad de Málaga \\ Manuel Ruíz Romero \\ Universidad de Sevilla
}

Recibido: 25/05/2016

Aceptado: 06/06/2016

\begin{abstract}
This article analyzes the political lines emanating from the educational legislation passed by the Andalusian government and the result of their application through some statistical data. This objective is of particular in terest because Andalusia becomes those years in the rehearsal for the educational policy of the Spanish socialism that will culminate with the approval of the LOGSE in 1990. They were mainly consulted and studied decrees, orders and resolutions regulatory system Andalusian and Spanish between 1982 and 1986. This work is accompanied by the necessary statistics to understand the context of this regulation. With these statistical and documen tary sou rces, it has been possible to analyze how from Andalusia understood and applied Artide 27 of the Constitution, with particular regard to the right to education and freedom of education. During these four years an Andalusian educational policy based on four main axes starts: ensuring access of citizens at all levels of the education system, promote Andalusian identity, improve the quality of education and ensure greater participation of the ed u cational community.
\end{abstract}

KEY WORDS: Educational policy, cultural policy, comparative education, illiteracy, adult education, decentralization, education system.

\begin{abstract}
RESUMEN
Este artículo pretende analizar las políticas públicas emanadas de la legislación educativa aprobada por los gobiernos andaluces durante la primera legislatura del Parlamento de Andalucía, y el resultado de su aplicación a través de algunos datos estadísticos. Este objetivo resulta de especial interés debido a que Andaluáa se convierte en esos años en el lugar de diseño y ensayo para la política educativa del socialismo español que tendrá su culminación con la aprobación de la LOGSE en 1990. Para ello, se han consultado y estudiado fundamentalmentelos decretos, órdenes y resoluciones reguladores del sistema andaluz y español entre 1982 y 1986. El trabajo se acompaña de las estadísticas necesarias para comprender el contexto de esta normativa. Con esas fuentes estadísticas y documentales, se ha podido analizar cómo desde Andaluća se entiende y aplica el artículo 27 de la Constitución, atendiendo especialmente al derecho a la educación y la libertad de enseñanza. Durante estos cuatro años se inicia una política educativa andaluza sustentada en cuatro ejes principales: garantizar el acceso de los ciudadanos a tod os los niveles de sistema educativo, fom entar la identidad andaluza, mejorar la calidad de la enseñanza y conseguir una mayor participación dela comunidad educativa.
\end{abstract}

PALABRAS CLAVE: Política de la educación, política cultural, educación comparada, analfabetismo, descentralización, educación de adultos, sistema educativo..

Dirección de correspondencia:

Manuel Hijano del Río, Departamento de Teoría e Historia de la Educación, Facultad de Ciencias de la Educación. Campus Teatinos. 29170, Málaga. e-mail: hijano@uma.es

Manuel Ruíz Romero, investigador del grupo HUM 618. Facultad de Comunicación. Departamento de Periodismo II. C/. Américo Vespucio s/n. 41092. Universidad de Sevilla. e-mail: mruizromero@ono.com 


\section{Introducción}

En Andalucía, el análisis de su proceso autonómico ha sido objeto de especial atención a lo largo de estos últimos quince años. Las peculiaridades de éste -como, por ejemplo, aprobar su Estatuto por la vía del artículo 151 de la Constitución de 1978- dotan a estos trabajos de un interés distinto al que pudiera suponer profundizar en esta cuestión en otras comunidades (Ruiz Romero, 2007).

El estudio de la política educativa en los años de la Transición cuenta con trabajos recientes y relevantes que nos han acercado a una realidad con unas significativas características. Así, debemos mencionar los de Juan Carlos Hernández Beltrán (2008), o los artículos de los monográficos sobre Transición democrática y educación de las revistas Historia de la Educación (González, 2002) y Educació i història (Mayordomo, 2011). Anterior a éstas, es destacable el monográfico de la revista Anales del Centro de Alzira de la UNED, dedicado a la educación en España entre 1978 y 1988 (Puelles, 1989).

Sin embargo, aunque el listado y calidad de dichos trabajos es extensa (Laudo, 2002), se considera que aún faltan estudios de índole autonómico que completen con detalle esa visión general. Sobre todo, si se tiene en cuenta que en la década de los ochenta en España se procede al cambio fundamental de administración y gobierno del sistema educativo, al pasar de una gestión totalmente centralizada a otra descentralizada a favor de las nuevas comunidades autónomas. Se conocen, para el caso andaluz, publicaciones elaboradas primordialmente con una metodología cuantitativa, desde una perspectiva sociológica en muchos casos, y apoyadas por el Gobierno de la Comunidad, tales como Delgado (1999), Requena y Cantón (2007) o la Consejería de Educación, Cultura y Deporte (2014). Estas publicaciones aportan unos datos estadísticos abundantes, muy relevantes y son una aproximación muy acertada a la realidad del sistema.

A estos trabajos generales, cabe añadir otros más puntuales, sobre aspectos específicos de la política educativa, tales como García, Criado y Cañal (2013), Hidalgo e Hijano (2011-12), Lassabille y Navarro (1997), Luengo, Luzón y Montes (1998), Pascual (1993), Mancila y Leiva (2011), o Sevilla (2001), entre otros.

Sin embargo, a estas referencias es aún necesario aportar un estudio político educativo general, de conjunto, que aborde las actuaciones del gobierno andaluz en esta materia tras asumir las competencias -a partir de 1982- y descubrir las líneas directrices que marcaron la ruta de inicio de la gestión. Son los años en los que se materializa el consenso constitucional conseguido en esta materia y el desarrollo normativo del conocido artículo 27 de la Constitución (Puelles, 2002). En palabras de Alejandro Mayordomo, "se remata la obra político-jurídica de la transición en el terreno educativo, regulando principios y objetivos que (...) se venían manifestando desde 1970" (Mayordomo, 2002, p. 47).

Ese es el objetivo fundamental de este trabajo: abordar, sistematizar y analizar principalmente desde la óptica legislativa, cuáles son los principios sobre los que se "(re)construye" la enseñanza en Andalucía en los primeros años de la Transición democrática. Como es bien sabido, el sistema andaluz reúne unas características políticas peculiares: cuantitativamente por su número de estudiantes y profesorado, el más importante de España y, además, el primero que asume el mayor grado de competencias en esta materia, dentro del marco de la Constitución de 1978, sin poseer un segundo idioma oficial (Caballero e Hijano, 2009). Igualmente, hay que añadir la extensión geográfica de su territorio donde la dispersión e importancia demográfica de sus municipios es muy significativa.

\section{Fechas importantes}

Superado el primero de los escollos del procedimiento para un autogobierno por la vía del artículo 151 de la Constitución, Andalucía necesita ahora la ratificación popular de su voluntad autonómica (Ruiz Romero, 2004). Para ello, se utiliza una Ley Reguladora de Referéndums que exige unas duras condiciones para superar satisfactoriamente esta consulta: el voto afirmativo de la mitad más uno de su censo electoral en cada una de sus ochos provincias. La mayoría centrista y 
socialista habían rechazado, durante la redacción de la citada norma, cualquier posibilidad de enmienda a esas rígidas condiciones presentadas por andalucistas y comunistas. Hasta tal punto que, pese a las advertencias estos, acerca de que si la suficiencia no se alcanzase en una sola provincia se condicionaba al resto, eso sería finalmente lo acontecido en Almería y por veinte mil votos. Además, UCD cuando la Ley va camino del Boletín Oficial del Estado, cambia su actitud inicial e invoca la abstención en el referéndum razonando su apuesta en favor de la vía del artículo y de estatus político del 143 de la Carta Magna: es decir, un autogobierno meramente administrativo.

Así pues, Andalucía pierde el referéndum del 28 de febrero de 1980 con la Ley en la mano. No obstante, la crispación y la movilización ciudadana son tan intensas, entre otras cuestiones ante los engaños del partido centrista y con el abierto boicot del ejecutivo de Suárez a la consulta que se precipita una salida jurídica y política al bloqueo. Aún a muy distinto ritmo y empuje, mediante diferentes iniciativas parlamentarias en Cortes acompañadas un respaldo/denuncia a lo acontecido desde las corporaciones locales y la junta preautonómica, las fuerzas parlamentarias pactan conjuntamente en octubre de 1980 un acuerdo político para desbloquear una situación que se había convertido en una crisis de Estado, cuando no es el principio del declive político del conductor de la Transición. Convalidados los resultados de febrero por la solicitud en dicho sentido de los parlamentarios almerienses, el proceso estatutario sigue su camino y la Asamblea de Parlamentarios Andaluces, reunida en Córdoba días después del intento de golpe de Estado del 23 de febrero de 1981, eleva su Proyecto articulado estatutario a las Cortes (Hijano y Ruiz Romero, 2001).

Despejado el procedimiento a seguir hacia la autonomía, el proceso andaluz continúa su marcha con normalidad institucional (Ruiz Romero, 2005). Finalizados los trámites que corresponden a toda ley básica del Estado, la norma comienza a ser aplicada. Las primeras elecciones autonómicas se celebran el 23 de mayo de 1982. La campaña da como resultado la mayoría absoluta del PSOE en el primer Parlamento de la historia de Andalucía (66 escaños del total de 109 con un 52,56 \% de votos); pero además, serviría de prólogo a los profundos cambios electorales que tendrían lugar en el Estado. El resto de formaciones que obtienen representación consiguen los siguientes escaños y porcentajes: UCD: 15 diputados (13,05\% del total de votos); PCA: 8 parlamentarios (8,53\%); y finalmente, los nacionalistas del PSA: 3 escaños (5,38\%). El 14 de junio de 1982 se constituye -aún sin sede propia- el primer Parlamento de Andalucía en el recinto del Alcázar hispalense. Comenzaba el periplo autonómico.

\section{El marco legal: Estatuto de Autonomía y las primeras transferencias}

Obviamente, las actuaciones en este periodo tienen su origen en dos documentos clave para entender lo acontecido posteriormente. Por un lado, el Estatuto de Autonomía y, por otro, el Decreto que aprueba el acuerdo de la Comisión Mixta de Transferencias de Andalucía (febrero, 1982).

El Estatuto de Autonomía Andaluz de 1981, en su artículo 19, fija como competencia en materia educativa de la Comunidad Autónoma "la regulación y administración de la enseñanza en toda su extensión niveles y grados, modalidades y especialidades en el ámbito de sus competencias", (Art. 19, LO 6/1981, de 30 de diciembre). La redacción de este artículo es prácticamente igual al artículo correspondiente al artículo 15 del Estatuto Catalán, al 16 del Vasco y al 31 del Gallego. Sólo en el texto andaluz, se añade un apéndice donde se solicita prestar especial atención a "las realidades, tradiciones, problemas y necesidades del pueblo andaluz".

El segundo documento mencionado, (RD 3936/1982, de 29 de diciembre) es mucho más complejo, detallado y sirve para conocer y evaluar en profundidad los límites jurídicos bajo los que se desenvuelve la futura Consejería de Educación.

Sin duda, este acuerdo reflejado en el Decreto, deja entrever la flexibilidad y amplitud de ambas partes negociadoras a la hora de desarrollar en el marco autonómico. El documento muestra cómo las autoridades educativas del momento empiezan a vislumbrar lo que supondría el futuro de la organización política sistema educativo español. Además, recoge no sólo la cesión de las competencias correspondientes a las Direcciones Provinciales, o de las Inspecciones Provinciales de 
Educación Básica del Estado, o las del Ministerio respecto a los centros privados; sino también la titularidad de los centros docentes de todos los niveles educativos no universitarios o las políticas de todo el personal funcionario del sistema.

A esta relación, se une la nada desdeñable competencia de la regulación de los niveles, grados, modalidades y especialidades de enseñanza y de normas y orientaciones pedagógicas. El acuerdo se completa con la relación de competencias reservadas para la "Administración Central del Estado" y la correspondiente valoración "provisional" de las cargas financieras de los servicios traspasados.

A partir de este momento, una vez conseguido el apoyo normativo suficiente en estos primeros años, prácticamente la totalidad de las actuaciones de la Consejería de Educación están dirigidas a la construcción o reforma del sistema educativo andaluz. En unos casos, para modificarlo de acuerdo con los nuevos tiempos democráticos y, en otros, para dar los primeros pasos encaminados a poner en marcha los ejes de una política educativa propia, con fines pedagógicos distintos a épocas anteriores.

\section{Inicios de una administración educativa andaluza}

Bajo este epígrafe, se encuadran las medidas que construyen una administración educativa andaluza. Entre noviembre de 1982 y a lo largo de 1983 el gobierno aprueba el primer organigrama de la primera Consejería de Educación andaluza. En ese momento, se dota de una Viceconsejería y tres Direcciones Generales (Ordenación Académica, Promoción Educativa y Renovación Pedagógica y Planificación y Financiación Educativa), además de una Secretaría General Técnica.

La relevancia del estudio de la estructura planteada trasciende del ámbito puramente organizativo o de gestión, puesto que esta normativa apunta las bases fundamentales de la actuación política del gobierno "encaminadas a la mejora de la calidad de la educación en la Comunidad Autónoma" (Art. 5 D 128/1982, de 13 de octubre). Desde ese momento, el concepto de calidad de la enseñanza se define a través de cuatro líneas fundamentales: la lucha contra el analfabetismo por medio de la educación de adultos, la formación y perfeccionamiento del profesorado, promover la investigación e innovación educativa y la compensación de las desigualdades sociales, geográficas en el ámbito educativo. En los años posteriores, son estas líneas las que definen la actuación política de la Consejería.

Efectivamente, en 1984, la Consejería concreta sus objetivos generales para planificar la enseñanza. Aprovechando la Ley Reguladora de los Consejos Escolares, en sus artículos 2 y 3, se detallan cuatro: por un lado, pretende conseguir el acceso de todos los andaluces a la educación para su realización personal como social, paliando las desigualdades; en segundo lugar, incrementar y fomentar la conciencia andaluza por medio de la difusión e investigación de sus señas de identidad: cultural, lingüística e histórica. Estos valores deben estar en estrecha relación con el entorno geográfico del centro. En tercer lugar, fomentar la participación de la comunidad educativa y, por último, "mejorar la calidad de la enseñanza en sus aspectos más esenciales" (LECE 4/1984, de 9 de enero).

El siguiente paso es la conversión de las Direcciones Provinciales de educación en Delegaciones Provinciales de la Consejería, a cuyo frente, el Delegado Provincial ejerce las competencias de representación, dirección y coordinación de la política de la Consejería en su territorio. (D 16/1983, de 26 de enero). En cada provincia solo sobreviven los "directores comisionados" cuya función es gestionar las escasas competencias aún vinculadas al Ministerio del ramo en Andalucía (RD 1223/1983, de 4 de mayo). Los Delegados Provinciales, designados directamente por el titular de la Consejería, son definidos como los ejecutores de una "labor trascendental en la gestión educativa".

A la desconcentración de funciones en el ámbito de las suministros y construcciones escolares, se le añaden posteriormente funciones decisorias sobre actividades extraescolares, comedores, transporte, educación compensatoria entre otros. También asumen competencias de 
interlocutores con los servicios centrales de la Consejería y para velar por el cumplimiento de la normativa aprobada (D. 269/1983, de 21 de diciembre).

En 1985 se considera como terminada la etapa de descentralización del sistema en Andalucía iniciada en los años preestatutarios. En esta Comunidad, la administración educativa ministerial queda integrada en el organigrama autonómico, con lo cual se confirma la tesis planteada por Foces Gil (2015) sobre la conformación de una administración periférica que justifica un periodo de transición. La Consejería de Educación andaluza pierde la oportunidad de buscar otro modelo organizativo bajo el principio de descentralización a favor de las provincias, de los centros educativos o incluso, tal y como se contempla en el Estatuto de Autonomía, de las comarcas. Se opta, sin embargo, por la copia del modelo de administración heredado de la década de los setenta, vigente aún hoy en día.

\section{Democratización del sistema educativo}

Ante la ausencia de una legislación marco sobre la participación y democratización de los centros escolares, el gobierno andaluz en la temprana fecha de octubre de 1982 regula por medio de un decreto el denominado Consejo Asesor de Educación. Este se concibe como "superior órgano consultivo y de participación de la comunidad educativa y de la sociedad andaluza en la Consejería de Educación" (Art. 1 D 104/1982, de 15 de septiembre).

Este Consejo nace para asesorar desde los primeros instantes de "(re)construcción" del sistema y cuenta con la participación de representantes del profesorado de los centros públicos y privados de todos los niveles no universitarios, de las familias y alumnos, de los directores de centros y titulares de los privados, de los sindicatos y, por último, personas de prestigio designadas por la Consejería.

La reorganización del sistema y su democratización también incluye a los centros. Como objetivo del gobierno, se plantea la necesidad de "crear las condiciones más convenientes que permitan una mayor eficacia en el funcionamiento de los centros docentes andaluces". (Preámbulo O 72/83 de 29 de agosto). El ejecutivo andaluz entiende la democratización de la enseñanza no sólo por medio de la participación de la comunidad educativa - "estamentos"- en los órganos colegiados del centro, sino también, así se menciona en la normativa, de la "autonomía" de los mismos.

Pero la iniciativa de estos años más relevante en este ámbito es la Ley de Consejos Escolares de 1984, cumpliendo con los artículos 27 de la Constitución y 12 del Estatuto de Autonomía. Su importancia se justifica porque en este texto, la Consejería asume la participación en la gestión del sistema como una aspiración de los sectores implicados, más "dinámicos", ya que se han heredado unos servicios burocráticos y pocos receptivos a los intereses de la sociedad. También el gobierno andaluz concreta el principio de democratización por medio de dos conceptos: representación y competencia. Entiende el primero como intervención e interlocución social para garantizar la vertebración de los colectivos y, el segundo, como medio para detallar y delimitar los ámbitos de cada sector (L 4/1984, de 9 de enero).

Otro aspecto reformado en este sentido y cuya repercusión va más allá de lo puramente simbólico, son los nombres de los centros; así como las nuevas direcciones de los mismos, cambiadas por los ayuntamientos democráticos. Se puede observar cómo la normativa regula no solo la asignación de códigos a cada centro, sino también sus denominaciones y callejero. En la normativa de estos años (1984-86) aparecen numerosos ejemplos de estos cambios. En agosto de 1983, se aprueba el calendario de evaluaciones de septiembre, el uso de los libros de escolaridad o el calendario escolar del curso 83/84 (O 70/1983 de 12 de agosto y R 53/1983 de 22 de junio) donde se otorga una gran "flexibilidad" a la hora de delimitar horarios de mañana o tarde, así como de inicio y finalización del curso.

También se acomete la labor de la formación constitucional de los estudiantes. En agosto de 1983 se prorroga lo establecido "en las disposiciones vigentes", completadas con la "especial 
atención" al "tema autonómico y en concreto al Estatuto de Andalucía" (O 72/1983 de 29 de agosto).

A partir de 1983, los centros docentes andaluces celebran el Día de la Constitución. La Consejería busca la implicación del profesorado a la hora de fomentar en los alumnos su conocimiento y respeto a los valores democráticos. Entre otras actividades, se destaca como obligatorio el izado de la bandera de España, junto a la de Andalucía (O 93/1983 de 16 de noviembre).

La formación constitucional se completa con el Programa de Cultura Andaluza, iniciado en esta primera legislatura. El Decreto regulador anuncia la necesidad de relacionar los contenidos de las enseñanza con las tradiciones y problemas de Andalucía. Para ello, es necesario dictar contenidos y objetivos educativos integrados en este Programa con la finalidad de "impulsar la búsqueda y promoción de todas las raíces de nuestra cultura", siempre y cuando este tratamiento se realice bajo la perspectiva de una comunidad histórica dentro de los pueblos de España (D 193/1984 de 3 de julio). Esta labor se completa con la creación de los Gabinetes Pedagógicos de Bellas Artes, encargados de transmitir el legado cultural y artístico andaluz. Conformado por docentes de varios niveles educativos, planificarán visitas y actividades pedagógicas en museos o lugares de interés del patrimonio (D 269/1985, de 26 de septiembre).

Este conjunto de medidas logran iniciar la democratización del sistema educativo andaluz. Se insertan los valores de la recién aprobada Constitución de 1978 en la vida cotidiana de las aulas por la vía del obligado cumplimiento de la legislación. Así, la educación andaluza a finales de la primera legislatura, ya cuenta con los órganos colegiados para la participación en su gestión y, por otro lado, los contenidos emanados de la Carta Magna empiezan a encontrar su sitio en todos los espacios y elementos educativos de un sistema que aún se encuentra regulado por una ley marco proveniente de la dictadura franquista (LGE).

\section{Renovación pedagógica}

Otro eje de la actuación política de la Consejería es la renovación pedagógica y la inclusión de métodos educativos innovadores, en un sistema educativo que cuenta con nuevas bases democráticas. Prueba del apoyo institucional, en este sentido, es creación de la ya mencionada Dirección General. Se asume la labor realizada desde 1970 por medio de los centros piloto o experimentales y se reconvierten en centros de régimen ordinario. Con ello se pretende que la innovación, renovación o transformación pedagógica no se circunscriba exclusivamente en unos centros determinados sino "la experimentación pedagógica y la innovación educativa podrá realizarse en cualquier centro no universitario de la comunidad autónoma de Andalucía", y que sea cualquier docente -individualmente o en equipo- quien realice un proyecto de innovación (Art. $2 \mathrm{D}$ 129/1983, de 22 de junio).

Meses más tarde, Andalucía se incorpora a la reforma del Ciclo Superior de la Educación General Básica y las Enseñanzas Medias (O 48/1984 de 2 de mayo). En un documento posterior, la Consejería evalúa la situación del Bachillerato y revela conclusiones extrapolables a todo el sistema: elevadas tasas de fracaso escolar, desmotivación y cansancio del profesorado, métodos pedagógicos no renovados, exceso de teoricismo en el Bachillerato, entre otros. En definitiva, el gobierno andaluz considera necesario plantear un nuevo sistema, mejor vinculado a la sociedad y la nueva realidad democrática. (Consejería de Educación y Ciencia, 1987).

En los primeros años, comienzan a trabajar los equipos de promoción y orientación educativa destinados a orientar y asesorar al profesorado; prevenir y disminuir las tasas de fracaso escolar, y orientar al alumnado tanto escolar como personalmente. Estos equipos pretenden clarificar y unificar las funciones de los múltiples servicios dedicados parcial o totalmente a esta labor en Andalucía. Hasta ese momento, coexisten servicios de orientación para el Curso de Orientación Universitaria (COU), servicios de orientación escolar y vocacional provinciales para EGB, equipos de orientación educativa familiar e institutos de orientación educativa y profesional. 
Todos ellos quedan integrados en estos nuevos equipos cuya finalidad se resume en tres aspectos: orientación al alumnado, apoyo al profesorado para el ejercicio de su labor y, por último, diagnóstico e integración del alumnado "en los casos que proceda" (D 238/1983, de 23 de noviembre).

Este ámbito de la innovación pedagógica cobra especial relevancia a la luz de los resultados obtenidos por el sistema educativo andaluz en estos años. Para ello, el gobierno andaluz ya manifiesta su preocupación por el fracaso escolar. Aunque dicho concepto ha variado notablemente en cuanto a su definición consensuada, y no se encuentran con facilidad las cifras correspondientes a lo que hoy se entiende como "abandono educativo temprano"; para este estudio se ha identificado "fracaso escolar" con la tasa obtención o no del graduado escolar para los estudiantes del último curso de la EGB. Así, se considera "fracaso" no conseguir el graduado escolar y, por tanto, sólo el "certificado de escolaridad".

En este sentido, y en comparación con la cifra española y las restantes comunidades autónomas, se observa cómo Andalucía está por encima de la media, aunque no llega a ocupar los últimos puestos. También se puede observar una gran dispersión de los datos, ya que nos encontramos tasas del 6\% para Navarra, frente a un 34\% de Asturias (Tabla 1)

Tabla 1.

Porcentaje de estudiantes de $8^{a}$ de EGB que obtienen certificado de escolaridad o graduado escolar al finalizar el curso 1986/87. Elaboración propia a partir de Ministerio de Educación y Ciencia (1989).

\begin{tabular}{lcc}
\hline & Graduado Escolar & Certificado de escolaridad \\
\hline España & 75,6 & 24,6 \\
Andalucía & 72,1 & 27,9 \\
Aragón & 81,5 & 18,5 \\
Asturias & 65,3 & 34,7 \\
Baleares & 76,9 & 23,1 \\
Canarias & 70,5 & 29,5 \\
Castilla Mancha & 77,2 & 22,8 \\
Castilla León & 86,7 & 13,3 \\
Cataluña & 71,4 & 28,6 \\
Cantabria & 82,7 & 17,3 \\
Valencia & 70,5 & 29,5 \\
Extremadura & 77,9 & 22,1 \\
Galicia & 73 & 27 \\
La Rioja & 85,7 & 14,3 \\
Madrid & 86 & 14 \\
Murcia & 80,1 & 19,9 \\
Navarra & 94,1 & 5,9 \\
País Vasco & 73,6 & 26,4 \\
Ceuta y Melilla & 76,9 & 23,1 \\
\hline
\end{tabular}

Pero donde se hace más hincapié desde la Consejería para la mejora del sistema educativo es en la formación permanente del profesorado. La preparación se basa en la revisión constante de su tarea y con la necesidad de adecuar la enseñanza a los nuevos conocimientos científicos, adecuándolos a las nuevas metodologías. Con el Programa de Formación y Perfeccionamiento del Profesorado de niveles no universitarios, el gobierno andaluz proporciona un marco de actuación único a las distintas experiencias habidas hasta ese momento, como son los Institutos de Ciencias de la Educación, Seminarios, Movimientos de Renovación Pedagógicas, entre otros. Este programa logra unificar esas iniciativas y fija unas categorías para su desarrollo (cursos, grupos de trabajo o reuniones) (O 54/1984 de 15 de mayo). 
Un punto de inflexión en este asunto es la creación de los Centros de Profesores. Redundando en los objetivos de convertir la educación andaluza en un sistema renovado al servicio de los valores democráticos, se plantea cambiar también la formación de los docentes por medio de una vía institucional que propicie intercambio de información pedagógica. Para este objetivo, se crean en 1985, al final de esta primera legislatura, los Centros de Profesores con la finalidad de convertirse en la "institución básica" del perfeccionamiento de los docentes. Esta labor se realiza por medio del intercambio de experiencias, reflexión sobre la enseñanza y fomento de programas de innovación.

La Consejería, con estas iniciativas, asume un papel muy relevante para coordinar y tutelar la renovación pedagógica en Andalucía, ya que con la creación de estos centros y la normativa mencionada, centraliza esta labor en la administración, no asumiendo otros modelos de formación e innovación basados más en la iniciativa de los equipos docentes o de los centros educativos (D) 16/1986, de 5 de febrero).

\section{Analfabetismo y educación de adultos}

Uno de los problemas más importantes de Andalucía en la década de los ochenta es la existencia aún de elevadas tasas de analfabetismo. Enmarcado en un nuevo tiempo democrático y con la experiencia de movimientos alternativos en la educación de adultos (Groves, 2016), la nueva administración educativa, desde octubre de 1983, diseña el denominado "Programa de Educación de Adultos de la Junta de Andalucía". Posteriormente, tras meses de experiencias piloto por medio del nuevo "Programa de Alfabetización y Educación de Adultos", la Consejería intenta coordinar a todas las instituciones implicadas bajo este conjunto de iniciativas. El Programa prevé unos planes interdepartamentales de la Junta, asesoramiento al profesorado para la elaboración de materiales didácticos específicos, fomentar la investigación en el ámbito de la educación de adultos y facilitar el paso de la alfabetización a la educación de adultos (O 80/1983, de 26 de septiembre).

El Programa se basa en los siguientes principios rectores (Consejería de Educación y Ciencia, 1991):

a) acción selectiva, frente a acción masiva. Se trata de seleccionar y subvencionar experiencias piloto que puedan a continuación ser implantadas en toda Andalucía.

b) nuevos planteamientos metodológicos. Implantación de un método global sintético frente al silábico.

c) formación del profesorado especializado en educación de adultos y preparado para trabajar en grupo.

d) municipalización del programa. Se trata de captar el apoyo de los ayuntamientos.

Posteriormente, se aprueba un nuevo diseño curricular con carácter experimental para el curso 85-86 en esta materia. Según sostiene la Consejería, la educación de adultos andaluza ha heredado "una estructura curricular poco realista, muy académica y excesivamente rígida" y esta circunstancia obliga a reformular estas enseñanzas. La Consejería busca un proyecto cercano a la realidad de los andaluces adultos y transformador de los objetivos, contenidos, estructura, evaluación, horarios, jornadas, agrupamientos, entre otros elementos. Concretamente este diseño nace con tres objetivos:

a) satisfacer las demandas de la sociedad

b) responder a las necesidades de los colectivos implicados en la enseñanza de adultos

c) y obtener un currículum adaptado a las situaciones reales de estos estudiantes.

Este diseño redefine la educación de adultos y la entiende como una labor transversal que comprende el desarrollo integral del individuo, la formación y capacitación laboral y la preparación para participar en la vida social para su transformación (O 119/1985 de 28 de noviembre). 
La realidad muestra lo acertado de la decisión, ya que el porcentaje de personas analfabetas en Andalucía es muy elevado en la década de los ochenta. (Tabla 2).

Tabla 2

Porcentajes analfabetismo en España en población mayor de 10 años (1970, 1981 y 1991). Elaboración propia a partir de Vilanova y Moreno (1992) y Ministerio de Educación y Ciencia (1992).

\begin{tabular}{lccc}
\hline & 1970 & 1981 & 1986 \\
\hline Andalucía & 15 & 12 & 10 \\
Aragón & 6 & 4 & 3 \\
Asturias & 4 & 2 & 2 \\
Baleares & 10 & 7 & 5 \\
Canarias & 13 & 8 & 7 \\
Cantabria & 2 & 2 & 1 \\
Castilla León & 4 & 3 & 2 \\
Castilla la Mancha & 15 & 11 & 10 \\
Cataluña & 6 & 5 & 4 \\
Extremadura & 14 & 12 & 10 \\
Galicia & 9 & 7 & 5 \\
Madrid & 4 & 3 & 3 \\
Murcia & 14 & 10 & 7 \\
Navarra & 3 & 2 & 2 \\
La Rioja & 4 & 3 & 2 \\
Valencia & 10 & 6 & 6 \\
País Vasco & 3 & 2 & 1 \\
España & 9 & 6 & 5 \\
\hline
\end{tabular}

Las cifras demuestran cómo efectivamente los esfuerzos políticos en cierta medida son recompensados con la disminución de la tasa de analfabetismo en Andalucía en este período (Tabla 2). Ahora bien, ese éxito en la gestión debe ser enmarcado en la tendencia general de todas las comunidades. Con esta tabla se puede observar la evolución de las cifras no sólo andaluzas, sino también de todas las comunidades y la general española. La conclusión es evidente: a pesar del descenso en las cifras, Andalucía, en 1986, aún permanece en el grupo de comunidades con mayor analfabetismo de España y no logra abandonar ese trío compartido con Extremadura y Castilla La Mancha donde los iletrados superan el 10\% de la población mayor de 10 años.

\section{Derecho a la educación}

Otro elemento significativo del incipiente sistema educativo de Andalucía es la enseñanza privada y, consecuentemente, la forma de entender el derecho y la libertad de educación por el gobierno socialista andaluz. A lo largo de 1983 se suceden órdenes reguladoras de la nueva situación administrativa, por medio de las cuales se autorizan "definitivamente" su apertura, en el marco de la aprobación del Decreto ley del gobierno central del 23 de agosto de 1980. (Hernández Beltrán, 2008). Aunque las referencias son numerosas, a modo de ejemplo, se puede mencionar la Orden de diciembre de 1984 en la que se alude a la "demanda social de puestos escolares" y a garantizar el derecho constitucional de las personas físicas y jurídicas de libertad de creación de centros docentes (O 5/85 de 5 de diciembre).

En Andalucía se entiende la necesidad de contar con estos centros, en cuanto a la presencia de la enseñanza privada en Andalucía, según la Tabla 3, resulta cercana en los niveles obligatorios, al 
$25 \%$ de los estudiantes. Se observa una presencia mayoritaria de la enseñanza pública, incluso muy superior a la media española a lo largo de estos años (1981-1986). Además, en estos años la educación primaria pública incrementa su representación en todos los niveles del sistema (Tablas 3 y 4).

Tabla 3

Porcentaje de estudiantes matriculados en la enseñanza primaria (EGB) pública (1981-1986). Elaboración propia a partir de: Ministerio de Educación y Ciencia (1989), Ministerio de Educación y Ciencia (1985) y Heredia y Parejo (2007).

\begin{tabular}{lccccc}
\hline & $1981 / 82$ & $1982 / 83$ & $1983 / 84$ & $1984 / 85$ & $1985 / 86$ \\
\hline España & 63,5 & 63,6 & 63,8 & 64,2 & 64,7 \\
Andalucía & 74,1 & 74,1 & 74,3 & 74,5 & 75,1 \\
\hline
\end{tabular}

Tabla 4

Porcentaje de estudiantes escolarizados en centros públicos/privados. Educación primaria. Andalucia (1981-1986). Elaboración propia a partir de: Consejo Escolar de Andalucia (2014).

\begin{tabular}{ccccc}
\hline Curso & Primaria & Infantil & Bachillerato & Formación Profesional \\
\hline $1981-82$ & 74,1 & 69,2 & 75,0 & 56,9 \\
$1982-83$ & 74,1 & 70,8 & 74,8 & 59,2 \\
$1983-84$ & 74,3 & 73,4 & 75,1 & 57,0 \\
$1984-85$ & 74,5 & 74,4 & 76,1 & 59,3 \\
$1985-86$ & 75,1 & 75,9 & 77,9 & 63,1 \\
\hline
\end{tabular}

La necesidad de extender la enseñanza pública en Andalucía, además obliga a la aprobación de normativa tanto en el sentido de evitar el absentismo escolar, como a la creación de "extensiones" de los Institutos de Bachillerato. Estas se convierten en el primer paso para acercar la enseñanza secundaria no obligatoria a las pequeñas poblaciones y, en paralelo, la Consejería requiere de la implicación de los ayuntamientos para erradicar el absentismo facilitando el transporte escolar, comedor y la Escuela Hogar. (D 253/1985, de 4 de diciembre y D 24/ 1986, de 12 de febrero).

Tras reseñar las actuaciones más destacadas de esta etapa inicial de la política educativa andaluza, cabe indicar cómo en el período tan limitado de estos cuatro años se ponen las bases de una programación general de la enseñanza, que perdurará en el tiempo. Esta acción de gobierno configura unos ejes sobre los que rotan las normas más importantes:

a) facilitar el acceso a todos los niveles del sistema educativo de todos los andaluces para compensar las desigualdades existentes y garantizar el ejercicio del derecho a la educación. Las medidas contra el analfabetismo y el apoyo a la educación de adultos son un buen ejemplo.

b) fomentar la conciencia de identidad andaluza, ensayando un modelo que no pretende la creación de un sentimiento nacionalista, sino al contrario, de pertenencia a Andalucía para la construcción de una España democrática.

c) ganar en participación y democratización del sistema educativo. La Consejería de Educación logra establecer un marco normativo que garantiza y regula la participación de los sectores de la comunidad educativa por medio de los consejos escolares.

d) garantizar una enseñanza de calidad como síntesis de los puntos anteriores. El gobierno andaluz ya en los años ochenta plantea la necesidad de incrementar no sólo cuantitativamente los indicadores o resultados del sistema, sino también mejorar cualitativamente su funcionamiento. 
La valoración y discusión de estas actuaciones adolecen de los necesarios estudios comparativos que podrían sin duda ampliar el campo de reflexión. A pesar de ello, se observa cómo a pesar de los esfuerzos realizados por el gobierno andaluz por superar los lastres de una herencia recibida, también en el ámbito educativo, las actuaciones podrían haber sido más audaces. Así, quedaron para otro momento una plena descentralización de la gestión del sistema, garantizar la autonomía plena de los equipos o movimientos de renovación pedagógica o la búsqueda de modelos alternativos para la lucha contra el analfabetismo. Sin embargo, se ha de valorar lo ingente de la labor acometida. Una administración compuesta en gran medida por políticos carentes de una larga trayectoria en la gestión política-educativa se vieron en cierto modo abrumados por la enorme responsabilidad de construir una estructura totalmente inédita en la Historia de la Educación de Andalucía y de España.

\section{Conclusiones}

Este trabajo demuestra el interés del estudio de la labor desarrollada por el primer gobierno autonómico andaluz (1982-1986). No sólo por las características propias de su sistema educativo ya enunciadas -cuantitativamente, el mayor de España y la primera comunidad autónoma donde se ejercen las competencias en educación sin tener un segundo idioma oficial y de las de mayor dispersión geográfica-, sino también porque Andalucía es el territorio donde dos realidades educativas históricas siguen siendo una realidad: el analfabetismo y el absentismo escolar. Estos condicionantes deben ser tenidos en cuenta a la hora de valorar la actuación política. El estado de la enseñanza que hereda la Junta de Andalucía, la Consejería de Educación, es paupérrimo. A falta de unos estudios más precisos sobre esta transición, se puede justificar que a pesar de los esfuerzos realizados hasta ese momento, con mayor o menor interés o intensidad por parte de las administraciones educativas, esta Comunidad aún tenía cifras más semejantes a las de "países en vías de desarrollo" que a países candidatos a la incorporación a la Comunidad Económica Europea.

En España se viven esos años convulsos de la Transición cuando el gobierno central está en manos de un partido en descomposición (UCD) en un periodo de interinidad, a la espera de unas elecciones generales (1982) que darán la victoria al Partido Socialista.

Con el contexto político de estos años, en Andalucía se pone en práctica el recién conseguido consenso constitucional en materia educativa. Es más, la aplicación de esa normativa por parte de un gobierno socialista (PSOE) con mayoría absoluta parlamentaria, se complementa con el necesario acuerdo con el resto de fuerzas parlamentarias y los representantes sociales de todas las tendencias de la comunidad educativa. Esta circunstancia exige la búsqueda de lugares intermedios especialmente en el ejercicio de los dos conceptos fundamentales, como el derecho a la educación o la libertad de enseñanza.

Por un lado, las fuerzas políticas y sociales de derecha/centro-derecha andaluzas, se ven obligadas a admitir el papel preponderante de la enseñanza pública. Así, las medidas adoptadas por el gobierno van encaminadas no sólo a incrementar la calidad de estas enseñanzas, entendida ésta desde la óptica "socialista", sino también a aumentar los porcentajes de estudiantes escolarizados en el sistema "estatal" en todos los niveles educativos. La implantación de un sistema regulador del acceso a la enseñanza sostenida con fondos públicos -es decir, incluida la concertada- es un ejemplo también de la cesión ideológica del entorno conservador.

Pero los partidos y fuerzas encuadradas en el ámbito de la izquierda/centro-izquierda, deben aceptar ese 25\% -aproximadamente- de presencia de la enseñanza privada laica/religiosa y la aplicación flexible del artículo 27 de la Constitución en cuanto a la libertad de creación de centros docentes. La normativa de estos años traduce la voluntad del gobierno andaluz de aceptar, aprobar y apoyar -incluso económicamente- la labor de estos colegios.

Andalucía es un escenario donde se ponen las bases de una política educativa socialista, que se traslada a todo el Estado a partir de la victoria del PSOE en las elecciones generales en octubre de 1982. La normativa sobre democratización del sistema o sobre el derecho a la educación 
demuestra cómo en esta Comunidad se aprueban medidas que, posteriormente, se adoptarán en todo el Estado.

Con un análisis cuantitativo, la evolución del número de disposiciones legales relevantes en materia educativa revelan dos periodos en los años analizados. Estos periodos coinciden con la dimisión del Rafael Escuredo (febrero 1984) y el nombramiento de Rafael Rodríguez de la Borbolla como presidentes andaluces. Es notorio cómo el segundo Presidente de la Junta impone un nuevo tempo legislativo, más pausado, a la acción de gobierno.

El análisis de la legislación y su ejecución demuestran el interés del gobierno del PSOE en aplicar sus principios ideológicos fundamentales en esta materia, basados en compensar desigualdades, la formación de los docentes y la renovación pedagógica.

El primer gobierno, en cuanto a la desigualdad en educación se refiere, debe atender a un territorio con altísimas tasas de analfabetismo y, por tanto, baja escolarización. También en este sentido, Andalucía representa un lugar distinto a otras comunidades, puesto que cuenta con sistema educativo público todavía en fase de crecimiento, donde no han llegado los esfuerzos de los años setenta para garantizar el ejercicio pleno del derecho a la educación. Durante este periodo, se logra con éxito aminorar la lacra del analfabetismo, aunque en paralelo con otras comunidades y aprovechando la tendencia iniciada diez años atrás. Las cifras demuestran cómo a finales de la década de los ochenta, aún Andalucía se encuentra entre las comunidades con más de un $10 \%$ de analfabetos entre su población de más de 10 años, duplicando la media española.

La renovación pedagógica es un aspecto esencial para en materia. Las nuevas libertades democráticas facilitan el desempeño en las escuelas de métodos educativos más acordes con la nueva sociedad española. Ya, en los años ochenta, habían surgido numerosos grupos de trabajo auspiciados o apoyados por diferentes administraciones o independientes- cuya labor innovadora comenzaba a sobresalir en Andalucía. La Consejería de Educación decide recoger todas esas iniciativas y, además, ampliar las posibilidades de trabajar en este sentido a la totalidad de los docentes andaluces. Se decide pasar del aislamiento, de la iniciativa deslavazada a la generalización. Otro aspecto desarrollado en este periodo es la formación permanente del profesorado. Son los años iniciales de un nuevo sistema de preparación de los docentes que aún continúa vigente hoy día.

Este conjunto de iniciativas centradas en el "cómo" enseñar, más que en los resultados, intentará trasladar los tiempos democráticos a la vida cotidiana del aula, a la labor del sistema educativo.

\section{Referencias bibliográficas}

Caballero Cortés, A., \& Hijano del Río. M. (2009). El analfabetismo en los discursos del Parlamento Andaluz desde la primera legislatura (1982-1986). En R. Berruezo Albéniz y S. Conejero López (eds.), El largo camino hacia una educación inclusiva: la educación especial y social del siglo XIX a nuestros días (pp. 381-390). Pamplona: Universidad Pública de Navarra.

Consejería de Educación y Ciencia, (1987). La reforma de las enseñanzas medias en Andalucía. Sevilla: Consejería de Educación y Ciencia.

Consejería de Educación y Ciencia, (1991). La educación de adultos en Andalucía. Sevilla: Consejería de Educación y Ciencia.

Consejería de Educación, Cultura y Deporte, (2014). 3 décadas autonomía educativa Andalucía (19832013) Informe sobre el estado y situación del sistema educativo en Andalucia. Sevilla: Consejería de Educación, Cultura y Deporte.

Consejo Escolar de Andalucía, (1993). Informe sobre el estado y situación del sistema educativo en Andalucia. 1983-1992. Huelva: Consejo Escolar de Andalucía. 
Delgado Valbuena, C. (1999). La educación primaria y secundaria en Andalucía. En E. Moyano Estrada y M. Pérez Yruela, Informe Social de Andalucia (1978-98). Dos décadas de cambio social, (pp. 189-222). Córdoba: CSIC e IESA.

Foces Gil, J. (2015). La Politica y administración de la educación en el estado autonómico (1978-2014). Desigualdades regionales y cohesión del sistema educativo. Estudio de un caso singular: Castilla y León. (Tesis doctoral). Universidad Nacional de Educación a Distancia.

García Carmona, A., Criado, A., \& Cañal de León, P. (2013). ¿Qué educación científica sugiere el currículo oficial de Andalucía para la etapa de Infantil? Investigación en la escuela, 79, 87-103.

Groves, T. (2016). Paulo Freire, la educación de adultos y la renovación pedagógica (1970-1983). Tendencias Pedagógicas, 27, 161-176.

Heredia, V. \& Parejo, A. (2007). Estadísticas históricas de educación y cultura en Andalucía. Sevilla: Instituto de Estadística de Andalucía.

Hernández Beltrán, J.C. (2008). Política y educación en la transición democrática española. Foro de educación, 10, 57-92.

Hidalgo González, A. \& Hijano del Río, M. (2011-12). El gasto público en educación del gobierno andaluz (2000-2008). Cuestiones pedagógicas. Revista de ciencias de la educación, 21,357-389.

Hijano del Río \& Ruiz Romero, M. (2001). Documentos para la bistoria de la autonomía andaluza (18821982). Málaga: Sarriá.

Instituto Nacional de Estadística. (1985). Estadística de la enseñanza en España, 1981/82. Madrid: Instituto Nacional de Estadística.

Lassibille, G. \& Navarro Gómez, M.L. (1997). Un análisis de los gastos privados de educación en Andalucía. Revista de Estudios Regionales, 49, 65-86.

Laudo, X. (2002). La educación y la transición democrática. Bibliografía. Historia de la Educación, 21, 307-310.

Luengo Navas, J.J., Luzón Trujillo, A., \& Montes Moreno, S. (1998). La educación infantil en Andalucía: de la LGE a la LOGSE. En V. Llorent Bedmar, Atención a la infancia y espacios educativos: aspectos comparados (pp. 543-560). Sevilla: Universidad de Sevilla.

Mancila, I. \& Leiva Olivencia, J.J. (2011). Revisión crítica de las políticas y prácticas educativas interculturales en Andalucía. En F.J. Sadio Ramos (coord.), Tendiendo puentes bacia la interculturalidad, (pp. 135-142). Granada-Málaga.

Mayordomo Pérez, A. (2002). La transición a la democracia: educación y desarrollo político. Historia de la Educación, 21, 19-47.

Mayordomo Pérez, A. (2011). L'educació a l'època de la transició a la democracia. Educació i historia, 18, 9-11.

Ministerio de Educación y Ciencia. (1989). Estadística de la Enseñanza en España, 1986/87. Madrid: Ministerio de Educación y Ciencia.

Ministerio de Educación y Ciencia. (1992). El sistema educativo español. Madrid: Ministerio de Educación y Ciencia.

Pascual Acosta, A. (1993). La educación de adultos en Andalucía. En J.A. Ortega Carrillo (coord.), La educación de adultos: situación actual y perspectivas de futuro (pp. 25-34). Madrid: Fundación Educación y futuro.

Puelles Benítez, M. (1989). Una década de política educativa (1978-1988). Anales del Centro de Alirira de la Universidad Nacional de Educación a Distancia, 5, 57-72.

Puelles Benítez, M. (2002). Pacto escolar constituyente: génesis, significación y situación actual. Historia de la Educación, 21, 49-66.

Requena, A. \& Cantón, F.J. (2007). El sistema educativo andaluzy su impacto social: un estudio longitudinal. Sevilla: Centro de Estudios Andaluces. Consejería de la Presidencia.

Ruiz Romero, M. (2004). El referéndum para la ratificación autonómica de Andalucia: el 28F como batalla mediática. Sevilla: Fundación Centro de Estudios Andaluces. Consejería de Presidencia.

Ruiz Romero, M. (2005). La conquista de la autonomía andaluza (1975-1982). Sevilla: IAAP.

Ruiz Romero, M. (2007). Guia bibliográfica sobre la Transición Andaluza. Sevilla: Fundación Centro de Estudios Andaluces. Consejería de Presidencia. 
Sevilla Merino, D. (2001). La educación infantil en Andalucía: logros y limitaciones de una política. Bordón, 3, 443-452.

Vilanova y Ribas, M. \& Moreno Julia, X. (1992). Atlas de la evolución del analfabetismo en España de 1887 a 1981. Madrid: Ministerio de Educación y Ciencia.

Legislación

Decreto 104/1982, de 15 de septiembre, por el que se crea el Consejo Asesor de Educación. Boletín Oficial de la Junta de Andalucía, de 2 de octubre de 1982, núm. 25. 399-401.

Decreto 128/1982, de 13 de octubre, por el que se desarrolla el Decreto 45/1982, de 4 de agosto, sobre estructura orgánica de la Consejería de Educación. Boletín Oficial de la Junta de Andalucía, 5 de noviembre de 1982, núm. 29. 450-452.

Decreto 16/1983, de 26 de enero, sobre Delegaciones de Educación de la Junta de Andalucía. Boletin Oficial de la Junta de Andalucia, de 4 de febrero de 1983, núm. 10. 86.

Decreto 129/1983, de 22 de junio, por el que se convierten en Centros de Régimen Ordinario los actuales Centros piloto creados al amparo del Decreto 2343/75 de 23 de agosto, y Bases para la Experimentación y la Innovación Pedagógica en los Centros de Régimen Ordinario en el marco territorial de competencias de la Comunidad Autónoma de Andalucía. Boletín Oficial de la Junta de Andalucía, de 8 de julio de 1983, núm. 54. 727-728.

Decreto 238/1983, de 23 de noviembre, por el que se crean los Equipos de Promoción y Orientación Educativa. Boletin Oficial de la Junta de Andalucia, de 3 de diciembre de 1983, núm. 98. 1406-1407.

Decreto 269/1983, de 21 de diciembre, sobre modificación de la estructura orgánica de la Consejería. Boletín Oficial de la Junta de Andalucia, de 10 de enero de 1984, núm. 3. 24-25.

Decreto 253/1985, de 4 de diciembre, por el que se amplía el Convenio con diversos ayuntamientos de la provincia de Málaga para la realización del programa de reducción del absentismo escolar. Boletín Oficial de la Junta de Andalucía, de 28 de enero de 1986, núm. 7. 256-257.

Decreto 269/1985, de 26 de septiembre, por el que se crean los Gabinetes Pedagógicos de Bellas Artes. Boletín Oficial de la Junta de Andalucia, de 12 de enero de 1986, núm. 5. 103.

Decreto 16/1986 de 5 de febrero, sobre creación y funcionamiento de los Centros de Profesores. Boletín Oficial de la Junta de Andalucia, de 21 de febrero de 1986, núm. 15. 486-488.

Decreto 24/1986, de 12 de febrero, por el que se regulan las extensiones de los Institutos de Bachillerato en la Comunidad Autónoma de Andalucía. Boletín Oficial de la Junta de Andalucía, de 18 de febrero de 1986, núm. 14. 440-441.

Ley Orgánica 6/1981, de 30 de diciembre, de Estatuto de Autonomía para Andalucía. Boletín Oficial del Estado, 11 de enero de 1982, núm. 9. 517-524.

Ley 4/1984, de 9 de enero, de Consejos Escolares. Boletín Oficial de la Junta de Andalucia, de 10 de enero de 1984, núm. 4. 47-49.

Orden de 9 de marzo de 1983, por la que se desarrolla el Decreto 128/1982, de 13 de octubre, sobre la estructura orgánica de la Consejería de Educación. Boletín Oficial de la Junta de Andalucia, de 15 de marzo de 1983, núm. 22. 200-203.

Orden de 12 de agosto de 1983, por la que se regula el calendario de evaluaciones del mes de septiembre y la utilización del libro de escolaridad en los centros de Educación General Básica. Boletín Oficial de la Junta de Andalucía, de 2 de septiembre de 1983, núm. 70. 929-930.

Orden de 29 de agosto de 1983, por la que se establecen normas básicas de organización y funcionamiento de los centros docentes no universitarios para el curso académico 1983-84. Boletín Oficial de la Junta de Andalucía, de 9 de septiembre de 1983, núm. 72. 984-985.

Orden de 16 de noviembre de 1983, de la Consejería de Educación, por la que se dan instrucciones para la celebración del Día de la Constitución en los centros docentes de Andalucía. Boletín Oficial de la Junta de Andalucía, de 22 de noviembre de 1983, núm. 93. 45. 
Orden de 26 de septiembre de 1983, por la que se desarrolla la Estructura Orgánica del Programa de Educación de Adultos de la Junta de Andalucía. Boletín Oficial de la Junta de Andaluciá, de 7 de octubre de 1983, núm. 80.1117-1118.

Orden de 2 de mayo de 1984, sobre la incorporación de la Comunidad Autónoma de And alucía al proceso de reforma del ciclo superior de Educación General Básica y de las Enseñanzas Medias. Boletín Oficial de la Junta de Andalucía, de 15 de mayo de 1984, núm. 48. 746.

Orden de 15 de mayo de 1984, por la que se establece el Programa de Formación y Perfeccionamiento del Profesorado de niveles no universitarios de la Comunidad Autóno ma de Andalucía. Boletín Oficial de la Junta de Andalucía, de 29 de mayo de 1984, núm. 54. 922-923.

Orden de 5 de diciembre de 1984, sobre modificaciones en Centros privados de Preescolar, Educación General Básica y Educación Especial en la Comunidad Autónoma de Andalucía. Boletín Oficial de la Junta de Andalucía, de 18 de enero de 1985, núm. 5. 80-81.

Orden de 28 de noviembre de 1985, por la que se autoriza a los centros de educación de adulto s de la Comunidad Autónoma de Andalucía a aplicar un nuevo diseño curricular, con carácter experimental, durante el curso 1985-86. Boletín Oficial de la Junta de Andalucia, de 14 de diciembre de 1985, núm. 119. 344.

Real Decreto 3936/1982, de 29 de diciembre, sobre traspaso de funciones y servicios de la Administración del Estado a la Comunidad Autónoma de Andalucía en materia de Educación. Boletín Oficial del Estado, 22 de enero de 1983, núm. 19. 1663-1664.

Real Decreto 1223/1983, de 4 de mayo, sobre medidas de reorganización de la administración periférica del Estado. Boletín Oficial del Estado, 17 de mayo de 1983, núm. 32. 13674.

Resolución de 22 de junio de 1983, de la Dirección General de Ordenación Académica, por la que se establecen criterios para la confección del Calendario Escolar en la Comunidad Autónoma de Andalucía. Boletín Oficial de la Junta de Andalucía, de 5 de julio de 1983, núm. 53. 722.

\section{Para citar este artículo}

Hijano del Río, M, \& Ruíz Romero, M. (2016). Descentralización educativa y comunidades autónomas: el caso andaluz (1982-1986). Revista Fuentes, 18(1), 49-63. [Fecha de consulta: dd $/ \mathrm{mm} / \mathrm{aa}$ ]. doi: doi: http://dx.doi.org/10.12795/revistafuentes.2016.18.1.03 